\title{
Bilayer paired quantum Hall states and Coulomb drag
}

\section{Citation}

Kim, Yong Baek, Chetan Nayak, Eugene Demler, N. Read, and S. Das Sarma. 2001. "Bilayer Paired Quantum Hall States and Coulomb Drag." Physical Review B 63 (20) (April 30). doi:10.1103/physrevb.63.205315.

\section{Published Version}

doi:10.1103/PhysRevB.63.205315

\section{Permanent link}

http://nrs.harvard.edu/urn-3:HUL.InstRepos:27990337

\section{Terms of Use}

This article was downloaded from Harvard University's DASH repository, and is made available under the terms and conditions applicable to Other Posted Material, as set forth at http:// nrs.harvard.edu/urn-3:HUL.InstRepos:dash.current.terms-of-use\#LAA

\section{Share Your Story}

The Harvard community has made this article openly available.

Please share how this access benefits you. Submit a story.

\section{Accessibility}




\title{
Bilayer paired quantum Hall states and Coulomb drag
}

\author{
Yong Baek Kim, ${ }^{1}$ Chetan Nayak, ${ }^{2}$ Eugene Demler, ${ }^{3}$ N. Read ${ }^{4}$ and S. Das Sarma ${ }^{5}$ \\ ${ }^{1}$ Department of Physics, The Ohio State University, Columbus, Ohio 43210 \\ ${ }^{2}$ Department of Physics, University of California, Los Angeles, California 90095 \\ ${ }^{3}$ Department of Physics, Harvard University, Cambridge, Massachusetts 02138 \\ ${ }^{4}$ Department of Physics, Yale University, P.O. Box 208120, New Haven, Connecticut 06520 \\ ${ }^{5}$ Department of Physics, University of Maryland, College Park, Maryland 20742
}

(Received 28 November 2000; published 30 April 2001)

\begin{abstract}
We consider a number of strongly correlated quantum Hall states that are likely to be realized in bilayer quantum Hall systems at total Landau level filling fraction $\nu_{T}=1$. One state, the $(3,3,-1)$ state, can occur as an instability of a compressible state in the large $d / l_{B}$ limit, where $d$ and $l_{B}$ are the interlayer distance and magnetic length, respectively. This state has a hierarchical descendent that is interlayer coherent. Another interlayer coherent state, which is expected in the small $d / l_{B}$ limit is the well-known Halperin $(1,1,1)$ state. Using the concept of composite fermion pairing, we discuss the wave functions that describe these states. We construct a phase diagram using the Chern-Simons Landau-Ginzburg theory and discuss the transitions between the various phases. We propose that the longitudinal and Hall-drag resistivities can be used together with interlayer tunneling to experimentally distinguish these different quantum Hall states. Our work indicates the bilayer $\nu_{T}=1$ quantum Hall phase diagram to be considerably richer than that assumed so far in the literature.

DOI: 10.1103/PhysRevB.63.205315

PACS number(s): 73.21.Ac, 71.10.Pm, 73.43.-f
\end{abstract}

\section{INTRODUCTION}

Bilayer quantum Hall systems at total Landau level filling factor $\nu_{T}=1$, i.e., $\nu=1 / 2$ in each layer, ${ }^{1}$ allow for novel interlayer coherent phases. These phases have attracted a great deal of theoretical and experimental attention ${ }^{2}$ over the last 16 years, dating back to a seminal paper by Halperin ${ }^{3}$ in which the multicomponent generalization of the Laughlin wave function was first considered in a rather general context. In particular, there is strong experimental evidence ${ }^{2,4}$ and compelling theoretical basis ${ }^{2,5}$ to believe that a spinpolarized bilayer $\nu=\frac{1}{2}$ quantum Hall system would have a novel spontaneous interlayer coherent incompressible phase for small values of the interlayer separation $d$, even in the absence of interlayer tunneling. (We consider only the situation without any interlayer tunneling in this paper, our considerations also apply to the physical situation with weak interlayer tunneling. The situation with strong interlayer tunneling is trivial by virtue of the tunneling-induced symmetric-antisymmetric single-particle tunneling gap that leads to the usual $\nu_{T}=1$ quantum Hall state in the symmetric band.) In the limit $d \rightarrow \infty$, however, one expects two decoupled layers each with $\nu=\frac{1}{2}$ and hence no quantized Hall state. The phase diagram for this compressible to incompressible quantum phase transition in $\nu=\frac{1}{2}$ bilayer systems has been studied extensively in the literature, ${ }^{2}$ but we still do not have a complete qualitative understanding of the detailed nature of this transition. In particular, one does not know how different kinds of incompressible (and compressible) phases compete as system parameters (e.g., $d$ ) are tuned, and how to distinguish among possible competing incompressible phases. In this paper, we revisit this issue by arguing that, in principle, there are several interesting and nontrivial quantum Hall phases in the $\nu_{T}=1$ bilayer system that could be systematically probed via interlayer drag experiments carried out at various values of the interlayer separation $d$. One goal of our paper is to describe and discuss the rich $\nu_{T}=1$ bilayer quantum Hall phase diagram using the Chern-Simons Landau-Ginzburg approach. ${ }^{6-10}$

Among the more interesting quantum Hall phases are the so-called paired Hall states, which have been extensively studied theoretically. ${ }^{3,11,12}$ In these states, the composite fermions form a superconducting paired state in which two composite fermions bind into effective Cooper pairs that condense into a ground state analogous to the BCS state. The well-studied Moore-Read Pfaffian state ${ }^{11}$ is a spin-polarized version of such a paired Hall state for a single-layer system. Bilayer paired Hall states have been discussed earlier in the literature in the context of $\nu=\frac{1}{2}$ (and $\nu=\frac{1}{4}$ systems), but no definitive idea has emerged regarding their experimental observability or their relation to the more intensively studied (and robust) $(1,1,1)$ state. ${ }^{2,3,5}$ The main purpose of the current paper is to critically discuss the possible existence of paired $\nu=\frac{1}{2}$ bilayer Hall states that, we argue, are distinguishable from the better-studied $(1,1,1)$ incompressible state (as well as from compressible states) through interlayer drag experiments. The transitions between these states are described by a variety of Landau-Ginzburg theories. Given the great current interest ${ }^{4,13}$ in the physics of $\nu=\frac{1}{2}$ bilayer systems, we believe that the results presented in this paper could shed considerable light on the nature of the possible quantum phase transitions in bilayer systems.

In Secs. II, III, and IV of this paper, we consider the possible bilayer $\nu=\frac{1}{2}$ quantum Hall phases in the parameter regimes $d \gg l_{B}$ (Sec. II), $d>l_{B}$ (Sec. III), and $d \leq l_{B}$ (Sec. IV), where $l_{B}$ is the magnetic length, which sets the scale for intralayer correlations. We argue that the likely ground states in these three regimes are, respectively, compressible (Fermi-liquid-like) states $\left(d \gg l_{B}\right)$, paired Hall states ( $d$ $\left.>l_{B}\right)$, and $(1,1,1)$ states $\left(d \sim l_{B}\right)$. In Sec. V, we discuss the transition between the $d>l_{B}$ and $d \leqslant l_{B}$ limits and introduce yet another state, a hierarchical descendent of the $(3,3,-1)$ 
state with interlayer coherence. We conclude in Sec. VI with a critical discussion of the various drag resistivities that we argue can, in principle, distinguish among these phases and could be used to study bilayer quantum phase transitions experimentally.

\section{II. $d \gg l_{B}$ : COMPRESSIBLE STATE}

Let us consider a bilayer system in which each layer has filling factor $\nu=1 / 2$ in the limit of the layer separation $d$ being much larger than the typical interparticle spacing, which is of the order of the magnetic length $l_{B}$. As a starting point, we will model the system by two almost independent Fermi-liquid-like compressible states, one in each layer. There are two alternative and complementary descriptions of compressible states at even-denominator filling fractions. We will briefly recapitulate some features of both as they will inform the following discussion of paired states.

One description of a Fermi-liquid-like compressible state at $\nu=1 / 2$ is based on the lowest Landau-level wave function $^{14}$

$$
\Psi_{1 / 2}\left(\left\{z_{i}\right\}\right)=\mathcal{P}_{L L L} \operatorname{Det} M \prod_{i<j}\left(z_{i}-z_{j}\right)^{2},
$$

where $M$ has the matrix elements $M_{i j}=e^{i \mathbf{k}_{i} \cdot \mathbf{r}_{j}}$. Here, $\mathbf{r}_{i}$ is the position of electron $i$. The $\mathbf{k}_{i} \mathrm{~s}$ are parameters that are chosen so that the total energy of the system is minimized. We will discuss their interpretation below. $\mathcal{P}_{L L L}$ projects states into the lowest Landau level; it has the following action: ${ }^{14,15}$

$$
\mathcal{P}_{L L L} e^{i \mathbf{k}_{i} \cdot \mathbf{r}_{j}} \mathcal{P}_{L L L}=e^{i \mathbf{k}_{i} \cdot \mathbf{R}_{j}},
$$

where $\mathbf{R}_{i}$ are the guiding-center coordinates of the electrons.

The corresponding wave function of the double-layer system at $\nu_{T}=1$ can be written as

$$
\Psi_{\text {compressible }}=\Psi_{1 / 2}\left(\left\{z_{i}^{\uparrow}\right\}\right) \Psi_{1 / 2}\left(\left\{z_{i}^{\downarrow}\right\}\right),
$$

where $\alpha=\uparrow, \downarrow$ label the two layers.

The lowest Landau-level constraint and Fermi statistics displace the electrons from the correlation holes, i.e., zeros of the wave function or, equivalently, vortices represented by the $\Pi_{i<j}\left(z_{i}-z_{j}\right)^{2}$ factor in the wave function. The Det $M$ factor is necessary to ensure Fermi statistics; it is a displacement operator ${ }^{16}$ because $\mathbf{k}_{i} \cdot \mathbf{r}_{j}$ acts as $\bar{k}_{i} z_{j}+k_{i} \partial / \partial z_{j}$ in the lowest Landau level. Thus the composite fermion made of an electron and two correlation holes has a dipolar structure. Using these ideas, the system can be described as a collection of dipolar "composite fermions" in which each dipolar fermion consists of an electron and the corresponding correlation holes. ${ }^{16-19}$ To each composite fermion we assign a $\mathbf{k}_{i}$ that is equal in magnitude (in units of $l_{B}$ ) and perpendicular to the displacement between the electron and its correlation holes. By Fermi statistics, the $\mathbf{k}_{i}$ s must be distinct. The structure of a dipolar composite fermion is given schematically in Fig. 1. The energy of a composite fermion increases with $\mathbf{k}_{i}$, so the ground state is a filled Fermi sea in $\mathbf{k}$ space. In the long-wavelength limit, the total energy of these dipolar com-

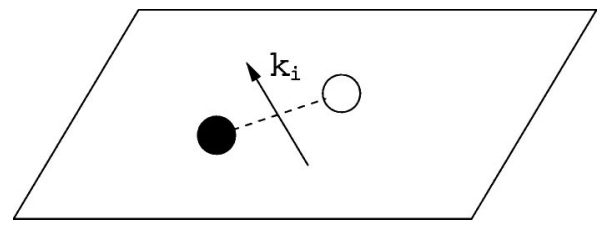

FIG. 1. Schematic picture of the dipolar composite fermion. The black and white dots represent the electron and vortex, respectively. The "wave vector" $\mathbf{k}_{i}$ is perpendicular to the dipole moment.

posite fermions can be approximately written as $\Sigma_{i} k_{i}^{2} / 2 m^{*}$ with effective mass $m^{*}$, which is determined by the interaction potential. ${ }^{17,19}$

An alternative formulation arises from the observation that an electron may be represented by a composite fermion together with a Chern-Simons gauge field that attaches two fictitious flux quanta to each fermion. ${ }^{20-22}$ This representation is mathematically equivalent to the original one but it naturally suggests another approximation. The composite fermions see zero average magnetic field due to the cancellation between the external magnetic field and the average fictitious magnetic field coming from the fictitious flux quanta. Consequently, the system can be described as an almost free (composite-) fermion system in zero effective magnetic field. In this approach, the fictitious flux quanta are introduced to represent the phase winding of the electron wave function around correlation holes associated with the positions of other electrons. The $\operatorname{Det}\left[e^{i \mathbf{k}_{i} \cdot \mathbf{R}_{j}}\right]$ factor in Eq. (1) can be interpreted as the wave function of the almost free composite-fermion system. ${ }^{14,22}$ Then $\mathbf{k}_{i}$ s can be regarded as the "kinetic momenta" of the composite fermions. In the long-wavelength, low-frequency limit, the two formulations are equivalent.

Note that in addition to the Fermi-liquid-like $\nu=1 / 2 \mathrm{com}$ pressible state in each layer one could have, in principle, other compressible states (e.g., charge-density wave or Wigner crystal) in the $d \gg l_{B}$ limit depending on the details of interaction and Landau-level coupling. In addition, strong disorder will lead to localized insulating states. We do not consider these possibilities in this paper.

\section{III. $d>l_{B}$ : PAIRING}

We will now implement the latter formulation in a double-layer system, and show that the compressible state has a strong pairing instability. ${ }^{12}$ We introduce two composite fermion fields $\psi_{\alpha}$ and two Chern-Simons gauge fields $\mathbf{a}_{\alpha}$, $\alpha=\uparrow, \downarrow$. The Hamiltonian is

$$
\begin{gathered}
H=H_{0}+H_{I}, \\
H_{0}=\int d^{2} r \sum_{\alpha=\uparrow, \downarrow} \frac{1}{2 m^{*}} \psi_{\alpha}^{\dagger}\left(\boldsymbol{\nabla}-\mathbf{a}_{\alpha}\right)^{2} \psi_{\alpha}, \\
H_{I}=\int d^{2} r \int d^{2} r^{\prime} \delta \rho_{\alpha}(\mathbf{r}) V_{\alpha \beta}\left(\mathbf{r}-\mathbf{r}^{\prime}\right) \delta \rho_{\beta}\left(\mathbf{r}^{\prime}\right)
\end{gathered}
$$

with the constraints $\boldsymbol{\nabla} \times \mathbf{a}_{\alpha}=2 \pi \widetilde{\phi} \delta \rho_{\alpha}(\mathbf{r}) . \widetilde{\phi}=2$ if the filling factor of each layer is $\nu=1 / 2$. Here $\delta \rho(\mathbf{r})=\rho(\mathbf{r})-\bar{\rho}$ is the 

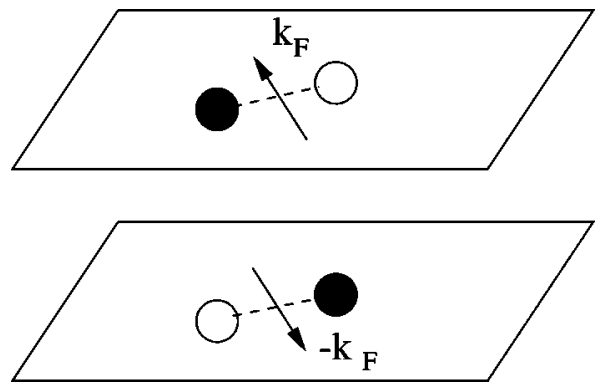

FIG. 2. Pairing of the "intralayer" composite fermions.

density disturbance measured from the average value $\bar{\rho}$. The interaction potential is given by $V_{\uparrow \uparrow}=V_{\downarrow \downarrow}=e^{2} / \varepsilon r$ and $V_{\uparrow \downarrow}$ $=V_{\downarrow \uparrow}=e^{2} / \varepsilon \sqrt{r^{2}+d^{2}}$ where $\varepsilon$ is the dielectric constant.

It is convenient to change the gauge field variables to $\mathbf{a}_{ \pm}=\frac{1}{2}\left(\mathbf{a}_{\uparrow} \pm \mathbf{a}_{\downarrow}\right)$. Then $H_{0}$ can be rewritten as

$$
\begin{aligned}
H_{0}= & \int d^{2} r\left[\frac{1}{2 m^{*}} \psi_{\uparrow}^{\dagger}\left(\boldsymbol{\nabla}-\mathbf{a}_{+}-\mathbf{a}_{-}\right)^{2} \psi_{\uparrow} \frac{1}{2 m^{*}} \psi_{\downarrow}^{\dagger}\right. \\
& \left.\times\left(\nabla-\mathbf{a}_{+}+\mathbf{a}_{-}\right)^{2} \psi_{\downarrow}\right]
\end{aligned}
$$

with $\boldsymbol{\nabla} \times \mathbf{a}_{ \pm}=\pi \widetilde{\phi}\left[\delta \rho_{\uparrow}(\mathbf{r}) \pm \delta \rho_{\downarrow}(\mathbf{r})\right]$. From Eq. (5), we see that $\psi_{\uparrow}$ and $\psi_{\downarrow}$ have the same gauge "charges" for $\mathbf{a}_{+}$but opposite gauge "charges" for $\mathbf{a}_{-}$. As a result, there will be an attractive interaction between the composite fermions in different layers via $\mathbf{a}_{-}$and a repulsive interaction via $\mathbf{a}_{+}$. Composite fermions in the same layer have repulsive interactions. As a result of Coulomb interactions, the attractive interaction mediated by the $\mathbf{a}_{-}$gauge field dominates in the low-energy limit and there exists a pairing instability between the composite fermions in different layers. This result can be understood in physical terms as follows. The $\mathbf{a}_{-}$and $\mathbf{a}_{+}$fields represent antisymmetric and symmetric density fluctuations. In the presence of Coulomb interactions, symmetric density fluctuations are highly suppressed but antisymmetric density fluctuations can still be large. As a result, the dynamic density fluctuations in the antisymmetric channel become more important in the low-energy limit and lead to a pairing instability.

This pairing instability has a natural explanation in the dipolar composite fermion picture. ${ }^{16-19}$ Let us take a dipolar composite fermion in layer $\uparrow$ with wave vector $\mathbf{k}^{\uparrow}=\mathbf{k}_{F}$ and a dipolar composite fermion in layer $\downarrow$ with wave vector $\mathbf{k}^{\downarrow}$ $=-\mathbf{k}_{F}$. As seen in Fig. 2, this configuration can lower the interlayer Coulomb energy because the electron in layer $\uparrow$ and the vortex in layer $\downarrow$ can sit on top of each other and vice versa.

This analysis predicts that, at least in principle, the Fermiliquid-like compressible state is always unstable to pairing. In practice, the pairing gap will be small in the limit $d \gg l_{B}$ and easily destroyed by disorder. As a result, we expect the pairing instability discussed above to be relevant for $d / l_{B}$ not too small. When $d / l_{B}$ becomes small, on the other hand, the starting point of two decoupled compressible states is no longer sensible and we take a different starting point, as described in later sections.
The wave function of the paired quantum Hall state constructed in this way can be written as

$$
\Psi_{\text {pair }}=\Psi_{\text {pair }}^{\mathrm{cf}} \prod_{i>j}\left(z_{i}^{\uparrow}-z_{j}^{\uparrow}\right)^{2} \prod_{k>l}\left(z_{k}^{\downarrow}-z_{l}^{\downarrow}\right)^{2},
$$

where

$$
\begin{aligned}
\Psi_{\text {pair }}^{\mathrm{cf}} & =\operatorname{Pf}\left[f\left(z_{i}, z_{j} ; \uparrow, \downarrow\right)\right] \\
& \equiv \mathcal{A}\left[f\left(z_{1}, z_{2} ; \uparrow, \downarrow\right) f\left(z_{3}, z_{4} ; \uparrow, \downarrow\right) \cdots\right],
\end{aligned}
$$

and $f\left(z_{1}, z_{2} ; \uparrow, \downarrow\right)$ is the pair wave function that depends on the symmetry of the pairing order parameter. $\operatorname{Pf}[\cdots]$ denotes the Pfaffian, which is defined in the second line, with $\mathcal{A}[\cdots]$ denoting the antisymmetrized product. Notice that $\Psi_{\text {pair }}$ can be regarded as the product of the wave function of the paired composite fermions and that of the $(2,2,0)$ bosonic Laughlin quantum Hall state.

It is not immediately clear what choice of $f\left(z_{1}, z_{2} ; \uparrow, \downarrow\right)$ is most favorable energetically. In the Chern-Simons theory of Ref. 12 there is a pairing instability in all angular momentum channels. ${ }^{12}$ In a modified Chern-Simons theory, it was claimed that the leading instability occurs in the $p$-wave channel. ${ }^{23}$ These approximate calculations do not necessarily capture the detailed energetics that determines the pairing symmetry. Hence, we will not enter into a discussion of energetics, but limit ourselves to a discussion of the simplest (and therefore likeliest) possibilities.

The simplest possibility is $p_{x}-i p_{y}$ pairing,

$$
\Psi_{\text {pair }}=\operatorname{Pf}\left[\frac{\uparrow_{i} \downarrow_{j}+\downarrow_{i} \uparrow_{j}}{z_{i}-z_{j}}\right] \prod_{i>j}\left(z_{i}^{\uparrow}-z_{j}^{\uparrow}\right)^{2} \prod_{k>l}\left(z_{k}^{\downarrow}-z_{l}^{\downarrow}\right)^{2} .
$$

Using the Cauchy identity,

$$
\prod_{i>j=1}^{N}\left(a_{i}-a_{j}\right)\left(b_{i}-b_{j}\right)=\prod_{i, j=1}^{N}\left(a_{i}-b_{j}\right) \operatorname{Det}\left|\left(a_{i}-b_{j}\right)^{-1}\right|,
$$

this can be rewritten as

$$
\Psi_{\text {pair }}=\Psi_{(3,3,-1)}=\prod_{i>j}\left(z_{i}^{\uparrow}-z_{j}^{\uparrow}\right)^{3}\left(z_{i}^{\downarrow}-z_{j}^{\downarrow}\right)^{3} \prod_{i, j}\left(z_{i}^{\uparrow}-z_{j}^{\downarrow}\right)^{-1} .
$$

Thus $\Psi_{\text {pair }}$ is the $(3,3,-1)$ state if one takes the $p_{x}-i p_{y}$ pairing. This wave function is well behaved in the longdistance limit but has a short-distance singularity. In the presence of Landau-level mixing, the short-distance part of the wave function can be modified without changing the structure of the wave function in the long-distance limit:

$$
\begin{aligned}
\Psi_{(3,3,-1)}= & \operatorname{Pf}\left[h\left(\left|z_{i}-z_{j}\right| / \xi\right) \frac{\uparrow_{i} \downarrow_{j}+\downarrow_{i} \uparrow_{j}}{z_{i}-z_{j}} \prod_{i>j}\left(z_{i}^{\uparrow}-z_{j}^{\uparrow}\right)^{2}\right. \\
& \times \prod_{k>l}\left(z_{k}^{\downarrow}-z_{l}^{\downarrow}\right)^{2} .
\end{aligned}
$$

Here, $h(0)=0$ and $h(x) \rightarrow 1$ as $x \rightarrow \infty$. In realistic systems, where Landau-level mixing is substantial, $\Psi_{(3,3,-1)}$ could be a good candidate for the paired quantum Hall state represented by $\Psi_{\text {pair }}$. It is natural to assume that $\Psi_{\text {pair }}$ does not 


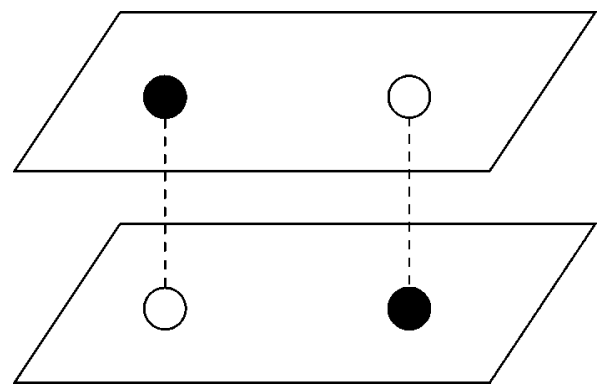

FIG. 3. The pairing of the "interlayer" composite fermions.

have an "interlayer Josephson effect" because there is no gapless neutral mode in the system, in contrast to the case of the $(1,1,1)$ state. $^{4,13}$ We will show this later by direct calculation.

Another possibility for the pair wave function is an exponentially decaying function with a correlation length $\xi$, for example, either of

$$
\begin{gathered}
\Psi_{\mathrm{SP}}=\operatorname{Pf}\left[e^{-\left|z_{i}-z_{j}\right| / \xi} \frac{\uparrow_{i} \downarrow_{j}+\downarrow_{i} \uparrow_{j}}{z_{i}-z_{j}}\right] \prod_{i>j}\left(z_{i}^{\uparrow}-z_{j}^{\uparrow}\right)^{2} \prod_{k>l}\left(z_{k}^{\downarrow}-z_{l}^{\downarrow}\right)^{2}, \\
\Psi_{\mathrm{SP}}=\operatorname{Pf}\left[e^{-\left|z_{i}-z_{j}\right| / \xi}\left(\uparrow_{i} \downarrow_{j}-\downarrow_{i} \uparrow_{j}\right)\right] \prod_{i>j}\left(z_{i}^{\uparrow}-z_{j}^{\uparrow}\right)^{2} \\
\times \prod_{k>l}\left(z_{k}^{\downarrow}-z_{l}^{\downarrow}\right)^{2} .
\end{gathered}
$$

This would correspond to a "strong" pairing (SP) state while the previous choice - the $(3,3,-1)$ state - corresponds to a "weak" pairing state in the terminology of Read and Green. ${ }^{24}$ The two different choices of SP wave functions (with $p$ - and $s$-wave pairs, respectively) in Eq. (12) can be continuously connected without crossing a phase transition.

\section{IV. $d \leqslant l_{B}:(1,1,1)$ STATE}

When the layer separation becomes sufficiently small, the interlayer Coulomb interaction can be comparable to or even larger than the intralayer Coulomb interaction. In this case, it should be more advantageous to first form an "interlayer" dipolar object that consists of an electron in one layer and two vortices in the other layer, then form a paired state of these "interlayer' composite fermions, as shown in Fig. 3. In the Chern-Simons formulation, this corresponds to the situation in which the electron in one layer can only see fictitious flux in the other layer. The appropriate ChernSimons constraint equations are

$$
\boldsymbol{\nabla} \times \mathbf{a}_{\uparrow}=2 \pi \widetilde{\phi} \delta \rho_{\downarrow}, \quad \nabla \times \mathbf{a}_{\downarrow}=2 \pi \widetilde{\phi} \delta \rho_{\uparrow}
$$

As in the previous case, we can form symmetric and antisymmetric combinations of the gauge fields $\mathbf{a}_{\uparrow}$ and $\mathbf{a}_{\downarrow}$. Again, the antisymmetric combination mediates an attractive interaction. The wave function of the corresponding paired quantum Hall state has the following form:

$$
\Phi_{\text {pair }}=\Phi_{\text {pair }}^{\mathrm{cf}} \prod_{i, j=1}^{N}\left(z_{i}^{\uparrow}-z_{j}^{\downarrow}\right)^{2},
$$

where

$$
\Phi_{\text {pair }}^{\mathrm{cf}}=\operatorname{Pf}\left[g\left(z_{i}, z_{j} ; \uparrow, \downarrow\right)\right]
$$

and $g\left(z_{1}, z_{2} ; \uparrow, \downarrow\right)$ is the appropriate pair wave function. Notice that the wave function $\Phi_{\text {pair }}$ can be regarded as the product of the wave function of a paired state of the composite fermions and that of the $(0,0,2)$ quantum Hall state for bosons.

However, this line of thinking appears to conflict with the conventional wisdom that a bilayer quantum Hall system at $\nu_{T}=1$ is described by the $(1,1,1)$ state for $d / l_{B} \sim 1 .^{3}$ Fortunately, the $(1,1,1)$ state

$$
\Psi_{(1,1,1)}=\prod_{i>j=1}^{N}\left(z_{i}^{\uparrow}-z_{j}^{\uparrow}\right)\left(z_{i}^{\downarrow}-z_{j}^{\downarrow}\right) \prod_{i, j=1}^{N}\left(z_{i}^{\uparrow}-z_{j}^{\downarrow}\right)
$$

can be rewritten in the form

$$
\Psi_{(1,1,1)}=\operatorname{Pf}\left[\frac{\uparrow_{i} \downarrow_{j}+\downarrow_{i} \uparrow_{j}}{z_{i}-z_{j}}\right]_{i, j=1}^{N}\left(z_{i}^{\uparrow}-z_{j}^{\downarrow}\right)^{2}
$$

using the Cauchy identity. In other words,

$$
g\left(z_{i}, z_{j} ; \uparrow, \downarrow\right)=\frac{\uparrow_{i \downarrow_{j}}+\downarrow_{i} \uparrow_{j}}{z_{i}-z_{j}}
$$

is the correct choice for $d / l_{B} \sim 1$.

Notice that this form of $g\left(z_{i}, z_{j} ; \uparrow, \downarrow\right)$ corresponds to the (pseudo-)spin triplet $p_{x}-i p_{y}$ pairing order parameter for the interlayer composite fermions. From this point of view, it is natural to have the same pairing symmetry (11) for $d>l_{B}$ but for intralayer rather than interlayer composite fermions.

\section{PHASE DIAGRAM AT $\nu_{T}=1$ : CHERN-SIMONS LANDAU-GINZBURG DESCRIPTION}

In this section, we consider these states within the framework of Chern-Simons effective field theories, ${ }^{6-10}$ the nature of the transitions between them, and also find an additional state that is a hierarchical descendent of the $(3,3,-1)$ state described by a $3 \times 3 \mathrm{~K}$ matrix.

The Lagrangian of the $(1,1,1)$ state is

$$
\begin{aligned}
\mathcal{L}^{(1,1,1)}= & \Psi_{\uparrow}^{\dagger}\left(i \partial_{t}+A_{\uparrow}^{0}-a_{\uparrow}^{0}-a_{\downarrow}^{0}\right) \Psi_{\uparrow}-\frac{1}{2 m} \mid \\
& \times\left.\left(\frac{\nabla}{i}+\mathbf{A}_{\uparrow}-\mathbf{a}_{\uparrow}-\mathbf{a}_{\downarrow}\right) \Psi_{\uparrow}\right|^{2}+\frac{1}{4 \pi} \epsilon^{\mu \nu \lambda} a_{\uparrow}^{\mu} \partial_{\nu} a_{\uparrow}^{\lambda} \\
& +(\uparrow \rightarrow \downarrow)-V_{i n t} .
\end{aligned}
$$

Here $\Psi_{\uparrow \downarrow}$ and $A_{\uparrow \downarrow}^{\mu}$ describe composite bosons and electromagnetic fields in the two layers, and the statistical gauge fields $a_{\uparrow \downarrow}^{\mu}$ ensure the agreement between this Lagrangian and the $(1,1,1)$ wave function. In the absence of interlayer tunneling, the number of electrons in each layer is conserved, so we can use the dual description ${ }^{25}$ of the $(1,1,1)$ state, 


$$
\begin{aligned}
\mathcal{L}_{d}^{(1,1,1)}= & \frac{1}{2}\left|\left(i \partial_{\mu}-b_{\mu}^{I}\right) \Phi_{v I}\right|^{2}+V\left(\Phi_{v I}\right)+\frac{1}{2}\left(f_{\mu \nu}^{I}\right)^{2} \\
& +\frac{1}{4 \pi} K_{I J} b_{\mu}^{I} \partial_{\nu} b_{\lambda}^{J} \epsilon^{\mu \nu \lambda}-\frac{1}{2 \pi} A_{\mu}^{a} t_{a}^{I} \partial_{\nu} b_{\lambda}^{I} \epsilon^{\mu \nu \lambda}
\end{aligned}
$$

Here $\Phi_{v I}$ describe vortices in the fields $\Psi_{I}$ with indices $I$ and $J$ labeling the layers $\uparrow$ and $\downarrow$, the dual gauge fields $b_{\mu}^{I}$ describe the conserved currents, the Greek indices $\mu, \nu, \lambda$ include space and time components, and the Gram (or $K$ ) matrix $^{8,9}$ is

$$
K_{I J}=\left(\begin{array}{ll}
1 & 1 \\
1 & 1
\end{array}\right) .
$$

It is also convenient to define the charge and spin gauge fields $A_{\mu}^{C, S}=\left(A_{\mu}^{\uparrow} \pm A_{\mu}^{\downarrow}\right) / 2, b_{\mu}^{C, S}=b_{\mu}^{\uparrow} \pm b_{\mu}^{\downarrow}$ with charge and spin vectors $\mathbf{t}_{C}=(1,1)$ and $\mathbf{t}_{S}=(1,-1)$, and $a=C, S$. Here and henceforth, we use the term "spin" to refer to the charge difference between the two layers, not the physical spin of the electrons, which is assumed to be fully polarized (i.e., spin here refers to an pseudospin associated with the layers).

A generic quasiparticle may now be constructed by taking a composite of $l_{1}$ vortices of type $\Phi_{v \uparrow}$ and $l_{2}$ vortices of type $\Phi_{v \downarrow} \cdot \Phi_{\left(l_{1}, l_{2}\right)}$ creates such a quasiparticle, which has charge $Q$ and $\operatorname{spin} S$

$$
\begin{aligned}
& Q=t_{C}^{T} K^{-1} l, \\
& S=t_{S}^{T} K^{-1} l .
\end{aligned}
$$

If the $K$ matrix has a vanishing determinant as it does in Eq. (21), then Eq. (22) will have to be modified. When this occurs, the zero eigenvalue corresponds to the Goldstone modes associated with some broken continuous symmetry. Hence, if we are to use the $K$-matrix formalism to calculate the quasiparticle properties and the degeneracy of the ground states on a torus [usually the degeneracy is det $K$ (Refs. 8 and 9)], some sort of reduced $K$ matrix will be required. We will now describe how this can be done in general. It is helpful to think in terms of the vectors in the condensate lattice ${ }^{8}$ the $K$ matrix is the Gram matrix of the lattice, that is, the matrix of inner products of a basis of vectors in the lattice. The inner product of two vectors $\mathbf{m}, \mathbf{n}$ in the lattice, represented as column vectors of integers [not to be confused with the similar vectors $l$, which lie instead in the dual (excitation) lattice], is then given by $\mathbf{m}^{T} K \mathbf{n}$. The vanishing determinant of $K$ implies that we can find a lattice vector $\mathbf{n}$ such that $K \mathbf{n}=\mathbf{0}$. Then the inner product of $\mathbf{n}$ with any other vector, including itself, is zero; we call $\mathbf{n}$ a null vector. We choose $\mathbf{n}$ to be primitive, that is, not divisible by any integer larger than 1 . Two vectors that differ by an integer multiple of $\mathbf{n}$ have the same inner product with any other vector because $\mathbf{n}$ is null. Hence, we can obtain a reduced lattice in which we identify vectors that differ by integer multiples of n and the inner product remains well defined. The reduced lattice is the quotient of the previous one by $\mathbf{n}$. In terms of matrices, the reduced $K$-matrix is obtained by changing basis, ${ }^{8,9}$ taking $\mathbf{n}$ as one of the basis vectors. Then in the resulting $K$-matrix, the entries in the row and column corresponding to $\mathbf{n}$ are all zero. The reduced $K$-matrix, $K_{\text {red }}$ is obtained by deleting this row and column. The process can be repeated until either a nonzero determinant is obtained or the lattice has dimension zero; in our examples, a single reduction is sufficient.

In the case of the $(1,1,1)$ state above, the null vector is $\mathbf{n}=(1,-1)$ and the reduced $K$ matrix is $K_{\text {red } I J}=(1)$, the same as in the polarized or single-layer $\nu=1$ state. The physical meaning of the procedure is that the $(1,1,1)$ state is obtained by condensing composite bosons with pseudospin or by taking a pseudospin-polarized state and tilting the pseudospins into the $X Y$ plane. This does not affect the quantum Hall properties of the state, which remain those of the single layer $\nu=1$ state. The procedure above correctly accounts for disregarding the direction of the pseudospin and implies that there is a single ground state on the torus up to low-lying states associated with the broken symmetry. The use of the reduced $K$ matrix gives the quasiparticle properties; the quasiparticles carry charge \pm 1 and are fermions. We see that the merons (vortices in the pseudospin order parameter that carry charge $\pm 1 / 2$ and ill-defined statistics) are not obtained from $K_{\text {red }}$, but are confined by the logarithmic potential between them, and cannot be separated to infinity with finite energy. Usually, the different degenerate ground states can be obtained from each other by creating a quasiparticle-quasihole pair, transporting one of them around the torus and subsequently annihilating them. The nontrivial statistics (Abelian, in all cases in this paper) of the quasiparticles then require degenerate ground states. Since the merons are confined, they do not contribute to the count of ground states, and indeed dragging one around the torus produces a helical texture in the ground state, increasing the energy by order width/length; we do not regard such a state as a ground state. In general, the ground-state degeneracy is divisible by the denominator of the filling factor $\nu_{T}$ (equal to 1 here); any ground-state degeneracy beyond that is not exact in a finite-size system, but the energy splitting $\sim \exp (-c L)$ on a torus of size $L$, where $c$ is a constant. Finally, spin wave states have excitation energies $\sim 1 / L$.

Returning to the dual Lagrangian in terms of the unreduced $K$ matrix of the $(1,1,1)$ state, in terms of the charge and spin gauge fields and the quasiparticle fields $\Phi_{(m, n)}$, it is

$$
\begin{aligned}
\mathcal{L}_{d}^{(1,1,1)}= & \frac{1}{2}\left|\left[i \partial_{\mu}-\left(\frac{m+n}{2}\right) b_{\mu}^{C}-\left(\frac{m-n}{2}\right) b_{\mu}^{S}\right] \Phi_{(m, n)}\right|^{2} \\
& +\frac{1}{4 \pi} b_{\mu}^{C} \partial_{\nu} b_{\lambda}^{C} \epsilon^{\mu \nu \lambda}-\frac{1}{2 \pi} A_{\mu}^{a} \partial_{\nu} b_{\lambda}^{a} \epsilon^{\mu \nu \lambda}+\frac{1}{2}\left(f_{\mu \nu}^{a}\right)^{2} .
\end{aligned}
$$

Since there is no Chern-Simons term for $b_{\mu}^{S}$, it is massless. This gauge field is dual to the Goldstone mode that results when $\Psi_{\uparrow}, \Psi_{\downarrow}$ condense, thereby breaking the U(1) pseudospin symmetry. Quantum fluctuations can disorder the pseudospin degree of freedom. This occurs when $\Phi_{(1,-1)}$ (the field for merons) condenses in Eq. (23). The effective theory for this transition is 


$$
\begin{aligned}
\mathcal{L}_{d}^{(1,1,1)}= & \frac{1}{2}\left|\left(i \partial_{\mu}-b_{\mu}^{S}\right) \Phi_{(1,-1)}\right|^{2}+V\left(\left|\Phi_{(1,-1)}\right|^{2}\right) \\
& +\frac{1}{4 \pi} b_{\mu}^{C} \partial_{\nu} b_{\lambda}^{C} \epsilon^{\mu \nu \lambda}-\frac{1}{2 \pi} A_{\mu}^{a} \partial_{\nu} b_{\lambda}^{a} \epsilon^{\mu \nu \lambda}+\frac{1}{2}\left(f_{\mu \nu}^{a}\right)^{2} .
\end{aligned}
$$

Applying $\mathrm{U}(1)$ duality $^{25}$ in reverse to the pseudospin alone, we find

$$
\begin{aligned}
\mathcal{L}_{d}^{(1,1,1)}= & \frac{1}{2}\left|\left(i \partial_{\mu}-A_{\mu}^{S}\right) \phi\right|^{2}+V\left(|\phi|^{2}\right)+\frac{1}{4 \pi} b_{\mu}^{C} \partial_{\nu} b_{\lambda}^{C} \epsilon^{\mu \nu \lambda} \\
& -\frac{1}{2 \pi} A_{\mu}^{C} \partial_{\nu} b_{\lambda}^{C} \epsilon^{\mu \nu \lambda}+\frac{1}{2}\left(f_{\mu \nu}^{a}\right)^{2} .
\end{aligned}
$$

The first line of Eq. (25) decouples from the second so the transition between the $(1,1,1)$ state and the quantum disordered state is in the $X Y$ universality class.

According to the arguments of Ref. 13, such a disordered state is the SP state of Eq. (12), with Landau-Ginzburg theory,

$$
\begin{aligned}
\mathcal{L}_{p}= & \Psi_{p}^{\dagger}\left(\partial_{0}-a_{0}-2 A_{0}^{C}\right) \Psi_{p}+\frac{1}{2 m}\left|\left[\vec{\partial}-i \vec{a}-2 i \vec{A}^{C}\right] \Psi_{p}\right|^{2} \\
& -\frac{1}{16 \pi} \epsilon_{\mu \nu \lambda} a_{\mu} \partial_{\nu} a_{\lambda} .
\end{aligned}
$$

The order parameter is given by $\Psi_{p}=\Psi_{\uparrow} \Psi_{\downarrow}$ [as opposed to $\Psi_{\uparrow, \downarrow}$ individually as in Eq. (19)]. Equation (26) may be derived from Eq. (19) in the limit that $\Psi_{p}$ is lighter than $\Psi_{\uparrow, \downarrow}$. The dual theory for SP is

$$
\begin{aligned}
\mathcal{L}_{d}^{S P}= & \frac{1}{2}\left|\left(i \partial_{\mu}-b_{\mu}^{C}\right) \Phi_{l}\right|^{2}+\frac{4}{4 \pi} b_{\mu}^{C} \partial_{\nu} b_{\lambda}^{C} \epsilon^{\mu \nu \lambda} \\
& -2 \frac{1}{2 \pi} A_{\mu}^{C} \partial_{\nu} b_{\lambda}^{C} \epsilon^{\mu \nu \lambda}+\frac{1}{2}\left(f_{\mu \nu}^{C}\right)^{2} .
\end{aligned}
$$

According to standard arguments, ${ }^{8,9}$ the SP ground states on a torus are fourfold degenerate and these ground states all have even electron number. ${ }^{24}$

We note the existence, in principle, of a state intermediate between the SP and $(1,1,1)$ states with the following Landau-Ginzburg theory

$$
\begin{aligned}
\mathcal{L}_{p}= & \Psi_{p}^{\dagger}\left(\partial_{0}-a_{0}-2 A_{0}^{C}\right) \Psi_{p}+\frac{1}{2 m}\left|\left[\vec{\partial}-i \vec{a}-2 i \vec{A}^{C}\right] \Psi_{p}\right|^{2} \\
& -\frac{i}{16 \pi} \epsilon_{\mu \nu \lambda} a_{\mu} \partial_{\nu} a_{\lambda}+\Psi_{s}^{\dagger}\left(\partial_{0}-2 A_{0}^{S}\right) \Psi_{s} \\
& +\frac{1}{2 m}\left|\left[\vec{\partial}-2 i \vec{A}^{S}\right] \Psi_{s}\right|^{2}+V\left(\left|\Psi_{p}\right|^{2},\left|\Psi_{s}\right|^{2}\right)
\end{aligned}
$$

which is valid when $\Psi_{p}=\Psi_{\uparrow} \Psi_{\downarrow}$ and $\Psi_{s}=\Psi_{\uparrow} \Psi_{\downarrow}^{\dagger}$ are light fields. The state in which $\left\langle\Psi_{p}\right\rangle \neq 0,\left\langle\Psi_{s}\right\rangle \neq 0$ also breaks pseudospin symmetry as a result of the latter order parameter. We will call this state SP/F to indicate the coexistence of distinct strong pairing and ferromagnetic order param- eters. Note that again the $K$ matrix for the SP state and the reduced $K$ matrix for the SP/F state are both just $K_{I J}=(4)$. The transition between the SP and SP/F states is an $X Y$ transition at which $\Psi_{s}$ condenses. The transition between the $\mathrm{SP} / \mathrm{F}$ and $(1,1,1)$ states is an Ising transition at which the symmetry $\Psi_{\uparrow, \downarrow} \rightarrow-\Psi_{\uparrow, \downarrow}$ is broken; it can also be viewed as a strong- to weak-pairing transition, similar to Ref. 24, but in the presence of ferromagnetic order in the pseudospin.

An alternative Chern-Simons Landau-Ginzburg theory yields the $(3,3,-1)$ state by Bose-condensing composite bosons that consist of an up electron and 3 vortices acting on the up electrons, and -1 vortices acting on the down electrons,

$$
\begin{aligned}
\mathcal{L}^{(3,3,-1)}= & \Psi_{\uparrow}^{\dagger}\left(i \partial_{t}+A_{\uparrow}^{0}-3 a_{\uparrow}^{0}+a_{\downarrow}^{0}\right) \Psi_{\uparrow}-\frac{1}{2 m} \mid \\
& \times\left.\left(\frac{\boldsymbol{\nabla}}{i}+\mathbf{A}_{\uparrow}-3 \mathbf{a}_{\uparrow}+\mathbf{a}_{\downarrow}\right) \Psi_{\uparrow}\right|^{2}+\frac{1}{4 \pi} \epsilon^{\mu \nu \lambda} a_{\uparrow}^{\mu} \partial_{\nu} a_{\uparrow}^{\lambda} \\
& +(\uparrow \rightarrow \downarrow)-V_{i n t} .
\end{aligned}
$$

As we have described in the previous section, this state can also be viewed as a paired state. Passing to the dual theory, we have Eq. (20), but with

$$
K_{I J}=\left(\begin{array}{rr}
3 & -1 \\
-1 & 3
\end{array}\right)
$$

In terms of the charge and spin gauge fields and the quasiparticle fields $\Phi_{(m, n)}$, the dual Lagrangian takes the form

$$
\begin{aligned}
\mathcal{L}_{d}^{(3,3,-1)}= & \mathcal{L}\left(\Phi_{(m, n)}\right)+\frac{1}{4 \pi} b_{\mu}^{C} \partial_{\nu} b_{\lambda}^{C} \epsilon^{\mu \nu \lambda}+\frac{2}{4 \pi} b_{\mu}^{S} \partial_{\nu} b_{\lambda}^{S} \epsilon^{\mu \nu \lambda} \\
& -\frac{1}{2 \pi} A_{\mu}^{a} \partial_{\nu} b_{\lambda}^{a} \epsilon^{\mu \nu \lambda}+\frac{1}{2}\left(f_{\mu \nu}^{a}\right)^{2}
\end{aligned}
$$

From Eq. (30) we can see that the charge sector of the $(3,3,-1)$ state is similar to the SP state. It has a quantized charge Hall conductance and supports elementary excitations of charge $1 / 2$. The pseudospin sector is different from that of either of the other states: it is gapped, unlike $(1,1,1)$, and exhibits a pseudospin Hall effect (which is manifested in the Hall drag resistance as we discuss later), unlike $(1,1,1)$ and SP.

The condensation of the neutral semion $(1,-1)$ in Eq. (31) eliminates the peudospin gauge field $b_{\mu}^{S}$ by the Anderson-Higgs effect, thereby leading to the SP state. This is analogous to the situation at $\nu_{T}=1 / 2$, where it was shown in Ref. 24 that the transition between the $(3,3,1)$ and strongpairing states is a second-order transition at which a Dirac fermion becomes massless. However in the $\nu_{T}=1$ case, we are dealing with a semion, rather than a fermion, so we might expect the transition to be analogous to the quantum Hall liquid to insulator transition. Both are described by a single relativistic field coupled to the Chern-Simons gauge field. In the large- $N$ limit this transition was shown to be second 
order, ${ }^{26,27}$ but in the relevant $N=1$ limit the gauge-field fluctuations may drive the transition first order. ${ }^{28}$ However, similar arguments in the absence of a Chern-Simons term were not conclusive. That transition was argued to be first order in $4-\epsilon$ dimensions, ${ }^{29}$ but three-dimensional duality ${ }^{25}$ implies that the transition is in the inverted $X Y$ universality class and, therefore, second order. Therefore, the possibility that the transition in the presence of the Chern-Simons term is second order appears to be still open. In the presence of disorder, at any rate, the transition will be second order. We believe, therefore, that the $\nu_{T}=1$ bilayer quantum phase transition between the $(3,3,-1)$ paired state and the SP state is a continuous phase transition.

We may, on the other hand, consider the condensation of the boson $(2,-2)$ upon the attachment of two flux quanta,

$$
\begin{aligned}
\mathcal{L}_{d}^{(3,3,-1)}= & \frac{1}{2}\left|\left(i \partial_{\mu}-2 b_{\mu}^{S}-\alpha_{\mu}\right) \widetilde{\Phi}_{(2,-2)}\right|^{2}+V\left(\left|\Phi_{2,-2}\right|^{2}\right) \\
& +\frac{1}{4 \pi} \alpha_{\mu} \partial_{\nu} \beta_{\lambda} \epsilon^{\mu \nu \lambda}+\frac{2}{4 \pi} \beta_{\mu} \partial_{\nu} \beta_{\lambda} \epsilon^{\mu \nu \lambda} \\
& +\frac{1}{4 \pi} b_{\mu}^{C} \partial_{\nu} b_{\lambda}^{C} \epsilon^{\mu \nu \lambda}+\frac{2}{4 \pi} b_{\mu}^{S} \partial_{\nu} b_{\lambda}^{S} \epsilon^{\mu \nu \lambda} \\
& -\frac{1}{2 \pi} A_{\mu}^{a} \partial_{\nu} b_{\lambda}^{a} \epsilon^{\mu \nu \lambda}+\frac{1}{2}\left(f_{\mu \nu}^{a}\right)^{2} .
\end{aligned}
$$

When $\widetilde{\Phi}_{2,-2}$ condenses, the resulting Meissner effect enforces the condition $2 b_{\mu}^{S}=\alpha_{\mu}$ (up to gauge transformations). Hence, the following quantum Hall state results:

$$
\begin{aligned}
\mathcal{L}= & \frac{4}{4 \pi} b_{\mu}^{S} \partial_{\nu} \beta_{\lambda} \epsilon^{\mu \nu \lambda}+\frac{2}{4 \pi} \beta_{\mu} \partial_{\nu} \beta_{\lambda} \epsilon^{\mu \nu \lambda}+\frac{1}{4 \pi} b_{\mu}^{C} \partial_{\nu} b_{\lambda}^{C} \epsilon^{\mu \nu \lambda} \\
& +\frac{2}{4 \pi} b_{\mu}^{S} \partial_{\nu} b_{\lambda}^{S} \epsilon^{\mu \nu \lambda}-\frac{1}{2 \pi} A_{\mu}^{a} \partial_{\nu} b_{\lambda}^{a} \epsilon^{\mu \nu \lambda}+\frac{1}{2}\left(f_{\mu \nu}^{a}\right)^{2},
\end{aligned}
$$

or

$$
\mathcal{L}=\frac{1}{4 \pi} K_{I J} b_{\mu}^{I} \partial_{\nu} b_{\lambda}^{J} \epsilon^{\mu \nu \lambda}+\frac{1}{2}\left(f_{\mu \nu}^{I}\right)^{2}-\frac{1}{2 \pi} A_{\mu}^{a} t_{a}^{I} \partial_{\nu} b_{\lambda}^{I} \epsilon^{\mu \nu \lambda}
$$

where $I, J=1,2,3, b_{\mu}^{3} \equiv \beta_{\mu}, t_{C}^{I}=(1,1,0), t_{S}^{I}=(1,-1,0)$, and

$$
K_{I J}=\left(\begin{array}{rrr}
3 & -1 & 2 \\
-1 & 3 & -2 \\
2 & -2 & 2
\end{array}\right) .
$$

This state is a hierarchical descendent of the $(3,3,-1)$ state (though the construction differs from the usual hierarchy by condensing neutral quasiparticles, which does not change the filling factor). A wave function for it can be constructed along the lines of Ref. 8. When the number of flux quanta on the sphere is $N_{\phi}=N-3$ [as in the $(3,3,-1)$ state], it contains two merons and two antimerons, which are bound in pairs to form two charge +1 excitations so the natural ground state has $N_{\phi}=N-1$. Also, it can occur for $N$ odd as well as for $N$ even, like the $(1,1,1)$ state and unlike the
$(3,3,-1)$ state. The state is distinct from the $(1,1,1)$ state, despite the fact that it breaks pseudospin symmetry (since $\widetilde{\Phi}_{(2,-2)}$ carries pseudospin, the same value as the electron) and has a gapless Goldstone mode. The $3 \times 3 \mathrm{~K}$ matrix has determinant zero and hence a reduced $K$ matrix is required. This can be obtained most easily by first making the basis change for the condensate lattice to basis vectors $(1,0,-1)$, $(0,1,1)$, and $(0,0,1)$ (relative to the previous basis). The resulting $K$ matrix is

$$
K_{I J}^{\prime}=\left(\begin{array}{lll}
1 & 1 & 0 \\
1 & 1 & 0 \\
0 & 0 & 2
\end{array}\right) .
$$

Since this contains the $(1,1,1)$ state $K$ matrix as a block, it is clear that the reduced $K$ matrix is

$$
K_{\text {red } I J}^{\prime}=\left(\begin{array}{ll}
1 & 0 \\
0 & 2
\end{array}\right) .
$$

Hence, the ground-state degeneracy on the torus is 2 . Incidentally, the block containing only 2 represents a "hidden $\mathrm{SU}(2)$ " in this state; the corresponding edge theory is an $\mathrm{SU}(2)$ current algebra at level 1, even though this will presumably not be a symmetry of the Hamiltonian.

We can complete the circle and return to our starting point, the $(1,1,1)$ state, if the quasiparticle $\Phi_{(1,-1,1)}$ (where the vortices are relative to the original basis) condenses in the state (34), thereby eliminating one of the neutral gauge fields by the Anderson-Higgs effect. The proliferation of these vortices leaves intact only those condensates (composite boson fields) that do not wind on going around these vortices. These condensates lie on a sublattice (of the unreduced lattice), which is the same as that of the $(1,1,1)$ state; in fact in the basis used for $K^{\prime}$ in Eq. (36) or for $K_{\text {red }}^{\prime}$ in Eq. (37), the transition has destroyed the condensate described by the $1 \times 1$ block at the lower right, leaving the $(1,1,1)$ state. This transition could be first order or second order in the absence of disorder according to the conflicting conventional wisdom discussed above.

A more useful form for the critical theory for the transition between $(3,3,-1)$ and its interlayer coherent descendent, along the lines of Eq. (25), may be derived from Eq. (32) by making the change of variables $\alpha_{\mu} \rightarrow \alpha_{\mu}-2 b_{\mu}^{S}$ and integrating out $\beta_{\mu}$. The Lagrangian takes the form

$$
\begin{aligned}
\mathcal{L}= & \frac{1}{2}\left|\left(i \partial_{\mu}-\alpha_{\mu}\right) \widetilde{\Phi}_{2,-2}\right|^{2}+V-\frac{1}{8 \pi} \alpha_{\mu} \partial_{\nu} \alpha_{\lambda} \epsilon^{\mu \nu \lambda} \\
& +\frac{1}{2 \pi} \alpha_{\mu} \partial_{\nu} b_{\lambda}^{S} \epsilon^{\mu \nu \lambda}+\frac{1}{2}\left(f_{\mu \nu}^{a}\right)^{2}+\frac{1}{4 \pi} b_{\mu}^{C} \partial_{\nu} b_{\lambda}^{C} \epsilon^{\mu \nu \lambda} \\
& -\frac{1}{2 \pi} A_{\mu}^{a} \partial_{\nu} b_{\lambda}^{a} \epsilon^{\mu \nu \lambda} .
\end{aligned}
$$

The gauge field $b_{\mu}^{S}$ only appears linearly in the Lagrangian, so we may integrate it out thereby resulting in the constraint that $\alpha_{\mu}=A_{\mu}^{S}$ up to a gauge transformation. The final Lagrangian is then 


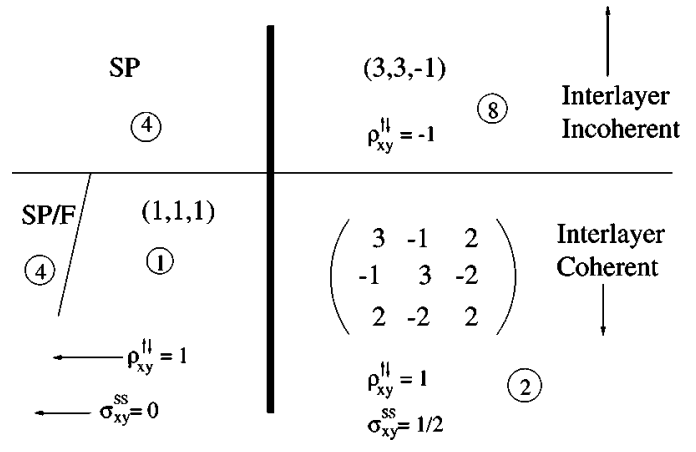

FIG. 4. Schematic phase diagram of states at $\nu_{T}=1$. The thick line represents a phase transition that may be first or second order (see text). The horizontal thin line represents a second-order phase transition in the $X Y$ universality class. The thin line separating the $\mathrm{SP} / \mathrm{F}$ and $(1,1,1)$ states is in the Ising universality class. The relationship between the states in this phase diagram is discussed in Sec. V. The drag resistivity, which together with interlayer tunneling, can distinguish these states is discussed in Sec. VI. The ground state degeneracies on the torus are encircled.

$$
\begin{aligned}
\mathcal{L}= & \frac{1}{2}\left|\left(i \partial_{\mu}-A_{\mu}^{S}\right) \widetilde{\Phi}_{2,-2}\right|^{2}+V\left(\left|\widetilde{\Phi}_{2,-2}\right|^{2}\right)-\frac{1}{8 \pi} A_{\mu}^{S} \partial_{\nu} A_{\lambda}^{S} \epsilon^{\mu \nu \lambda} \\
& +\frac{1}{2}\left(f_{\mu \nu}^{I}\right)^{2}+\frac{1}{4 \pi} b_{\mu}^{C} \partial_{\nu} b_{\lambda}^{C} \epsilon^{\mu \nu \lambda}-\frac{1}{2 \pi} A_{\mu}^{C} \partial_{\nu} b_{\lambda}^{C} \epsilon^{\mu \nu \lambda} .
\end{aligned}
$$

Hence, the transition between the $(3,3,-1)$ state and its interlayer coherent hierarchical descendent is also in the $X Y$ universality class.

These states and transitions are depicted in the phase diagram of Fig. 4. The two states in the upper portion of the phase diagram - SP and $(3,3,-1)-$ are not interlayer coherent while the three states in the lower portion $[\mathrm{SP} / \mathrm{F}$, $(1,1,1)$, and the interlayer coherent descendent of $(3,3,-1)$ (identified by its $K$ matrix)] are interlayer coherent [i.e., they spontaneously break pseudospin U(1) symmetry]. The development of interlayer coherence may be probed by interlayer tunneling experiments. ${ }^{4}$ The states on the right in Fig. 4 are expected for $d \gg l_{B}$ while those on the left are expected for $d \sim l_{B}$. As we discuss in the next section, these may be distinguished by their Hall drag resistivities. This phase diagram suggests that the transition between the $(3,3,-1)$ and $(1,1,1)$ states may occur via an intermediate state that is either the SP state or the interlayer coherent hierarchical descendent of the $(3,3,-1)$ state. We caution the reader that first-order transitions between any of these states are possible, even those that are not adjacent in the figure. However, the $X Y$ transitions can be second order.

\section{DRAG RESISTIVITIES}

It is important to discuss experiments that can distinguish the $\Psi_{(1,1,1)}$ state, the $\Psi_{(3,3,-1)}$, its interlayer coherent descendent, and the SP state. Here, we propose Coulomb-drag experiments in which the longitudinal, $\rho_{x x}^{\uparrow \downarrow}$ and Hall, $\rho_{x y}^{\uparrow \downarrow}$, drag resistivities are used to distinguish the different phases. These may be calculated in Chern-Simons theory from

$$
\rho_{i j}^{\alpha \beta}=\rho_{i j}^{\mathrm{cf}} \alpha \beta+\epsilon_{i j} \rho^{\mathrm{cs} \alpha \beta},
$$

where $i, j=x, y, \alpha, \beta=\uparrow, \downarrow, \epsilon_{i j}$ is the antisymmetric tensor, and $\rho^{\mathrm{cs} \alpha \beta}=2 \delta_{\alpha \beta}$ in the compressible and $(3,3,-1)$ states (intralayer composite fermions) while $\rho^{\mathrm{cs} \alpha \beta}=2 \sigma_{\alpha \beta}^{x}$ in the $(1,1,1)$ state (interlayer composite fermions).

First, consider the longitudinal drag resistivity. In the compressible state, if we neglect gauge-field fluctuations, $\rho_{x x}^{\text {cf }} \uparrow \downarrow=0$, so $\rho_{x x}^{\uparrow \downarrow}=0$. Including these fluctuations, it vanishes as $T^{4 / 3}$ at low temperatures. ${ }^{30}$ In $\Psi_{(1,1,1)}$ and $\Psi_{(3,3,-1)}$ (as well as its interlayer coherent descendent), $\rho_{x x}^{\text {cf }} \uparrow \downarrow$ and, hence $\rho_{x x}^{\uparrow \downarrow}$ vanish at zero temperature and are activated at low temperatures. ${ }^{31}$

Now, let us consider the Hall drag resistivity. In the compressible state, both terms on the right-hand side of Eq. (40) vanish so the Hall drag resistivity vanishes. In the $(3,3$, -1) state, $\rho_{x y}^{\mathrm{cf}}$ is that of a $p_{x}+i p_{y}$ superconductor, which has vanishing charge resistivity (since it is a superconductor) but quantized spin Hall resistivity. ${ }^{24,33}$ In other words, $\rho_{x y}^{\text {cf } c c}=0, \rho_{x y}^{\text {cf } c s}=0, \rho_{x y}^{\text {cf } s s}=1$. Consequently, $\rho_{x y}^{\uparrow \uparrow}=\rho_{x y}^{\downarrow \downarrow}=3$ and $\rho_{x y}^{\uparrow \downarrow}=-1$.

In the $(1,1,1)$ state, $\rho_{x y}^{\text {cf }}$ is identical but $\rho_{x y}^{\text {cs }}$ is different so $\rho_{x y}^{\uparrow \uparrow}=\rho_{x y}^{\downarrow \downarrow}=1$ and $\rho_{x y}^{\uparrow \downarrow}=1$, in agreement with Ref. 32. The same result can be deduced physically by noting that interlayer coherence requires that the voltage be the same in both layers. If we run a current in one layer alone, then this condition can only be satisfied if $\rho_{x y}^{\uparrow \uparrow}=\rho_{x y}^{\uparrow \downarrow}$. On the other hand, the total Hall resistance of the system is $\left(\rho_{x y}^{\uparrow \uparrow}+\rho_{x y}^{\uparrow \downarrow}\right) / 2$. Since this must equal 1 , we obtain the previously stated result. Note that the same logic applies to the interlayer coherent descendent of the $(3,3,-1)$ state that must, therefore, have $\rho_{x y}^{\uparrow \uparrow}=\rho_{x y}^{\uparrow \downarrow}=1$. In other words, the full resistivity tensor of the $(1,1,1)$ state is identical to that of the interlayer coherent descendent of the $(3,3,-1)$ state.

Note that the interlayer coherent descendent of the $(3,3$, -1) state discussed in the previous section is a pseudospin Hall superconductor. From Eq. (39), we see that the pseudospin conductivity tensor is of the form

$$
\sigma^{S S}=\left(\begin{array}{cc}
\frac{\kappa}{i \omega} & \frac{1}{2} \\
-\frac{1}{2} & \frac{\kappa}{i \omega}
\end{array}\right),
$$

where $\kappa$ is a constant. Hence, there is nonvanishing spin Hall conductivity. However, upon inverting this tensor, we see that the spin Hall resistivity vanishes, as it must in order to satisfy $\rho_{x y}^{\uparrow \uparrow}=\rho_{x y}^{\uparrow \downarrow}=1$. The $(1,1,1)$ state, on the other hand, has vanishing spin Hall conductivity. The distinction between a pseudospin Hall superconductor and an "ordinary" pseudospin superconductor is reminiscent of the difference between a Hall insulator and an ordinary insulator (but inverted).

Thus far, we have focused on the situation in which the layers are perfectly balanced. If they are unbalanced due to the presence of an external bias field, for example, this is analogous to introducing a pseudospin Zeeman field along 
the $z$ direction. This will have a pair-breaking effect on the paired states and will be expected to weaken the quantum Hall effect. This should, as a consequence, increase the longitudinal drag (along with the total longitudinal resistance). The presence of external bias can thus be used to distinguish the paired state from other incompressible states.

We conclude by summarizing our results. We have shown that the $\nu=\frac{1}{2}\left(\nu_{T}=1\right)$ bilayer quantum Hall system (in the absence of interlayer tunneling) is likely to have as its ground state a novel paired Hall state (possibly of $p$-wave symmetry) for intermediate layer separations $d>l_{B}$, which gives way to the usual $(1,1,1)$ state for smaller layer separations $\left(d \leqq l_{B}\right)$, and to compressible Fermi-liquid-type states (two decoupled Halperin-Lee-Read ${ }^{22} \nu=\frac{1}{2}$ layers) for large layer separations $\left(d \gg l_{B}\right)$. We argue that the quantum phase transitions separating the paired states from the $(1,1,1)$ and bilayer Halperin-Lee-Read states can be experimentally studied via the measurement of various components of interlayer drag resistivities. We also argue that the transition between the $(3,3,-1)$ and $(1,1,1)$ states may occur via an intermediate state that is either the SP state or the interlayer coherent hierarchical descendent of the $(3,3,-1)$ state and in either case one of the two transitions will be in the $X Y$ universality class.

\section{ACKNOWLEDGMENTS}

We would like to thank L. Balents, J. Eisenstein, and S. Sachdev for discussions. Y.B.K., C.N., N.R., and S.D.S. would like to thank the Aspen Center for Physics for hospitality. This work was supported by the NSF under Grant Nos. DMR-9983783 (Y.B.K.), DMR-9983544 (C.N.), DMR9818259 (N.R.); the A.P. Sloan Foundation (Y.B.K. and C.N.); the Harvard Society of Fellows (E.D.); and the ONR (S.D.S.).
${ }^{1}$ Throughout this paper, we use $\nu_{T}$ to denote the total filling factor and $\nu$ to denote the filling factor in each layer, $\nu_{T}=2 \nu$.

${ }^{2}$ See, for example, the articles by J. P. Eisenstein, S. M. Girvin, and A. H. MacDonald, in Perspectives in Quantum Hall Effects, edited by S. Das Sarma and A. Pinczuk (Wiley, New York, 1997), and references therein.

${ }^{3}$ B. I. Halperin, Helv. Phys. Acta 56, 75 (1983); Surf. Sci. 305, 1 (1994)

${ }^{4}$ I. B. Spielman, J. P. Eisenstein, L. N. Pfeiffer, and K. W. West, cond-mat/0002387 (unpublished).

${ }^{5}$ X. G. Wen and A. Zee, Phys. Rev. Lett. 69, 1811 (1992); Phys. Rev. B 47, 2265 (1993).

${ }^{6}$ S. C. Zhang et al., Phys. Rev. Lett. 62, 82 (1989).

${ }^{7}$ N. Read, Phys. Rev. Lett. 62, 86 (1989).

${ }^{8}$ N. Read, Phys. Rev. Lett. 65, 1502 (1990).

${ }^{9}$ B. Blok and X.-G. Wen, Phys. Rev. B 42, 8133 (1990); 42, 8145 (1990); 43, 8337 (1991).

${ }^{10}$ K. Moon et al., Phys. Rev. B 51, 5138 (1995).

${ }^{11}$ G. Moore and N. Read, Nucl. Phys. B 360, 362 (1991).

${ }^{12}$ N. E. Bonesteel, Phys. Rev. B 48, 11484 (1993); N. E. Bonesteel, I. A. MacDonald, and C. Nayak, Phys. Rev. Lett. 77, 3009 (1996); M. Greiter, X.-G. Wen, and F. Wilczek, ibid. 66, 3205 (1991).

${ }^{13}$ E. Demler, C. Nayak, and S. Das Sarma, cond-mat/0008137 (unpublished).

${ }^{14}$ E. Rezayi and N. Read, Phys. Rev. Lett. 72, 900 (1994); 73, 1052 (1994)

${ }^{15}$ S. M. Girvin and T. Jach, Phys. Rev. B 29, 5617 (1984).

${ }^{16}$ N. Read, Semicond. Sci. Technol. 9, 1859 (1994); Surf. Sci. 361, 7 (1996).

${ }^{17}$ R. Shankar and G. Murthy, Phys. Rev. Lett. 79, 4437 (1997); D. H. Lee, ibid. 80, 4745 (1998); V. Pasquier and F. D. M.
Haldane, Nucl. Phys. B 516, 719 (1998).

${ }^{18}$ B. I. Halperin and A. Stern, Phys. Rev. Lett. 80, 5457 (1998); A. Stern et al., Phys. Rev. B 59, 12547 (1999).

${ }^{19}$ N. Read, Phys. Rev. B 58, 16262 (1998).

${ }^{20}$ J. K. Jain, Phys. Rev. Lett. 63, 199 (1989); Adv. Phys. 41, 105 (1992).

${ }^{21}$ A. Lopez and E. Fradkin, Phys. Rev. B 44, 5246 (1991); V. Kalmeyer and S.-C. Zhang, ibid. 46, 9889 (1992).

${ }^{22}$ B. I. Halperin, P. A. Lee, and N. Read, Phys. Rev. B 47, 7312 (1993).

${ }^{23}$ T. Morinari, Phys. Rev. Lett. 81, 3741 (1998).

${ }^{24}$ N. Read and D. Green, Phys. Rev. B 61, 10267 (2000).

${ }^{25}$ M. Peskin, Ann. Phys. (Belgium) 113, 122 (1978); P. O. Thomas and M. Stone, Nucl. Phys. B 144, 513 (1978); C. Dasgupta and B. I. Halperin, Phys. Rev. Lett. 47, 1556 (1981); X. G. Wen and A. Zee, Int. J. Mod. Phys. B 4, 437 (1990); M. P. A. Fisher and D. H. Lee, Phys. Rev. B 39, 2756 (1989).

${ }^{26}$ X. G. Wen and Y. S. Wu, Phys. Rev. Lett. 70, 1501 (1993).

${ }^{27}$ X. G. Wen, Phys. Rev. Lett. 84, 3950 (2000).

${ }^{28}$ L. Pryadko and S. C. Zhang, Phys. Rev. Lett. 73, 3282 (1994).

${ }^{29}$ S. Coleman and E. Weinberg, Phys. Rev. D 7, 1888 (1973); B. I. Halperin, T. C. Lubensky, and S.-K. Ma, Phys. Rev. Lett. 32, 292 (1974).

${ }^{30}$ Y. B. Kim and A. J. Millis, Physica E (Amsterdam) 4, 171 (1999); cond-mat/9611125 (unpublished); I. Ussishkin and A. Stern, Phys. Rev. B 56, 4013 (1997); S. Sakhi, ibid. 56, 4098 (1997).

${ }^{31}$ G. Vignale and A. H. MacDonald, Phys. Rev. Lett. 76, 2786 (1996); I. Ussishkin and A. Stern, ibid. 81, 3932 (1998); F. Zhou and Y. B. Kim, Phys. Rev. B 59, R7825 (1999).

${ }^{32}$ K. Yang, Phys. Rev. B 58, R4246 (1998).

${ }^{33}$ T. Senthil and M. P. A. Fisher, Phys. Rev. B 60, 4245 (1999). 